\section{Evaluación de la frecuencia de espina bífida oculta y su posible relación con el arsénico ambiental en una muestra prehispánica de la Quebrada de Camarones, norte de Chile}

VERÓNICA SILVA-PINTO $^{\text {a }}$, BERNARDO ARRIAZA ${ }^{2,3,4 b}$, VIVIEN STANDEN $^{1 \mathrm{c}}$

\section{Spina bifida occulta associated with environmental arsenic exposure in a prehispanic sample from northern Chile}

Background: The Camarones River Valley, located in the extreme north of Chile, is characterized by high environmental arsenic levels and an arid desert. It has been inhabited by humans for the past 7,000 years. Evidence exists for chronic arsenic poisoning in both prehispanic and present populations residing in the area. Chronic arsenic exposure causes multi-systemic problems and can induce congenital malformations, in particular neural tube development defects such as spina bifida. Aim: To study the prevalence of spina bifida among prehispanic mummies of the area. Material and Methods: One-hundred and twenty prehistoric adult individuals were analyzed for evidence of spina bifida occulta of the sacrum in skeletal samples from the sites of Camarones 8, Camarones 9, Azapa 140 and Lluta 54, held in repository at the Museo Universidad de Tarapacá de Arica-San Miguel de Azapa. A diagnosis was considered positive when at least S1, S2 or S3 were affected. As controls, mummies of individuals that resided in Lluta and Azapa valley, with a low arsenic exposure, were analyzed. Results: The frequency of spina bifida occulta among samples from the Camarones coast and Lluta and Azapa Valley were 13.5 and 2.4\% respectively. Conclusions: Considering these were contemporaneous samples, and are believed to have had no other differences in diet or other factors, the differential exposures to arsenic could have produced the observed differences in spina bifida frequencies.

(Rev Med Chile 2010; 138: 461-469).

Key words: Arsenic; Mummies; Spina bifida occulta.

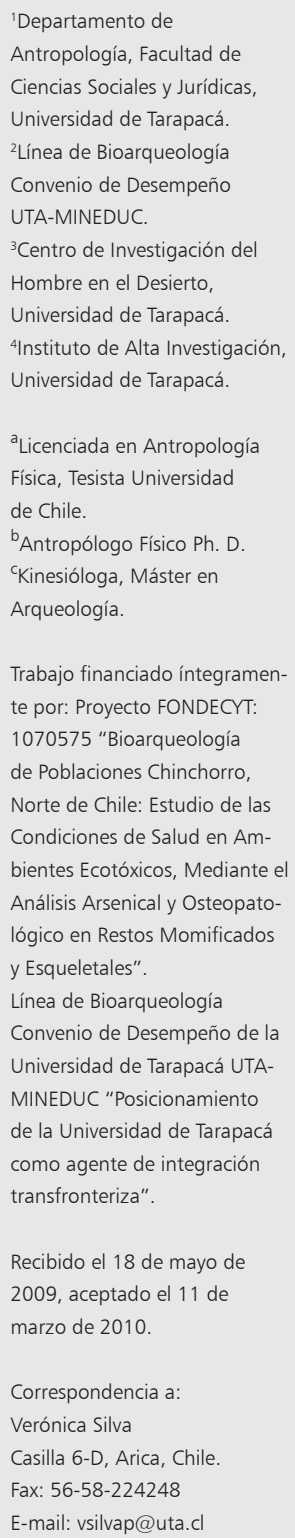

$\mathrm{H}$ oy en día sabemos, gracias a estudios epidemiológicos, que existen un sinnúmero de contaminantes ambientales que acarrean desfavorables consecuencias a la salud de las poblaciones afectadas, muchos de los cuales son producto de la acción antrópica: la actividad minera, industrial, agrícola, pesquera y otras áreas de producción; a las que se suman sustancias tóxicas de origen natural, dentro de las cuales el metaloide arsénico es uno de los más comunes $^{1-4}$. En Sudamérica las zonas más afectadas por la presencia de arsénico corresponden al norte chileno, noroeste argentino, sur peruano y suroeste boliviano, entre los paralelos $14^{\circ}$ y $28^{\circ}$ Sur. Este metaloide se origina en la cordillera de Los Andes, encontrándose principalmente en el agua, tanto superficial como profunda, con una dispersión definida y focalizada aunque disemi- 
nada regionalmente y con concentraciones muy variables ${ }^{1,3-5}$.

El norte de Chile, entre los $17^{\circ} 30^{\prime}$ y $28^{\circ} 30^{\prime}$ S, tiene un área de aproximadamente $250.000 \mathrm{~km}^{2}$, de los cuales $35.000 \mathrm{~km}^{2}$ corresponden a rocas cuaternarias, existiendo también en esta zona $420 \mathrm{vol}-$ canes, algunos de los cuales se encuentran activos ${ }^{6}$. Una de las áreas con más altos niveles de arsénico en Chile corresponde a la quebrada de Camarones, ubicada en el extremo norte, en la XV Región de Arica y Parinacota, caracterizada por su ambiente marcadamente desértico y ecotóxico, con niveles de arsénico en el agua que varían según la época del año y el sector a lo largo del valle, en un rango de 1.000 a $5.100 \mu \mathrm{g} \mathrm{L}^{-1}$, encontrándose además, en sustratos del suelo y en vegetales cultivados de comunidades rurales que utilizan el agua del río para regadío. Estos valores son muy superiores a los $10 \mu \mathrm{g} \mathrm{L}^{-1}$ de arsénico en agua de bebida que establece la Norma Chilena 409/1.Of2005 ${ }^{7}$, en concordancia con los límites establecido por la OMS y la Unión Europea a través de la Directiva 98/83/EC ${ }^{8,9}$. Otros valles de la región presentan arsénico, pero en niveles considerablemente menores (Tabla 1).

La ruta más común de ingreso del arsénico al organismo humano es a través del agua de bebida, el consumo de alimentos contaminados y la inhalación por vía respiratoria ${ }^{1,2}$, ocasionando en altas dosis daño multisistémico, afecciones a la piel, desórdenes vasculares periféricos, neuropatías, anemia, hepatomegalia, hiperpigmentación, diarreas crónicas e incrementando el riesgo a contraer cáncer ${ }^{3,10,11}$. Además, trae consecuencias a la reproducción humana, ya que puede inducir abortos espontáneos, muerte perinatal, bajo peso al nacer y malformaciones congénitas ${ }^{12-13}$.

La etapa de desarrollo embrionario de mayor susceptibilidad frente a la acción de agentes terató-

Tabla 1. Niveles de concentración de arsénico en los valles de la Región de Arica y Parinacota ${ }^{6}$

\begin{tabular}{|lcc|}
\hline Área & $\begin{array}{c}\text { Arsénico } \\
\text { en agua }\end{array}$ & $\begin{array}{c}\text { n de veces que } \\
\text { sobrepasa la } \\
\text { norma }\end{array}$ \\
\hline Lluta & $280 \mathrm{a} 30 \mu \mathrm{g} \mathrm{L}^{-1}$ & $28-3$ \\
Azapa & $20 \mu \mathrm{g} \mathrm{L}^{-1}$ & 2 \\
\hline Camarones & 1.000 a $5.100 \mu \mathrm{g} \mathrm{L}^{-1}$ & $100-500$ \\
\hline
\end{tabular}

genos, corresponde a los períodos somítico y prefetal, entre las $3^{\text {a }}$ y $8^{\mathrm{a}}$ semanas, cuando se produce la organogénesis ${ }^{14}$. Según los modelos propuestos, el arsénico inorgánico cruza la barrera placentaria y se acumula selectivamente en el neuroepitelio, incidiendo directamente en el desarrollo de malformaciones congénitas, especialmente relacionadas con el cierre del tubo neural ${ }^{15-19}$.

La espina bífida es el más común de los defectos del tubo neural, se produce por una interrupción en el proceso de cierre del tubo que se completa el día 28 de gestación, con una causa preponderantemente multifactorial y un significativo componente ambiental ${ }^{20}$. En general su prevalencia en la actualidad es baja, la tasa observada en ECLAMCChile (Estudio Colaborativo Latinoamericano de Malformaciones Congénitas) durante el período 1969 -1999, fue de 8,61 por cada 10.000 nacimientos $(0,086 \%)^{21-22}$. Desde el año 2000 en Chile se fortifican las harinas de trigo con ácido fólico, lo que ha disminuido a la mitad la tasa de espina bífida en comparación con períodos previos a la fortificación ${ }^{23}$.

En paleopatología la gran mayoría de los casos reportados de espina bífida en contextos arqueológicos corresponden a espina bífida oculta en el área sacra, con una alta prevalencia de $5 \%$ a $25 \%$ en $S 1^{24,25}$. La espina bífida oculta sacra en contextos bioarqueológicos ha sido en general asociada a grupos endogámicos y a aislamiento biocultural; esta explicación se presenta en los trabajos de Ferembach ${ }^{26}$ para una población de Taforalt en Marruecos, con una data de 10070 a 8500 a.C. que presenta una ocurrencia de espina bífida oculta cercana a $33 \%$; Rodríguez- Martin ${ }^{27}$ reporta que $30 \%$ de la población guanche de Tenerife en Islas Canarias presenta espina bífida; Mas-Pascual et $\mathrm{al}^{28}$ para la población de Hierro, también en Islas Canarias, reporta una ocurrencia de $28,7 \%$.

Para población prehispánica del valle de Azapa en Arica y de Ica en Perú, Gerszten et $\mathrm{al}^{29}$ reportan una ocurrencia de espina bífida oculta de $92 \%$, así mismo Allison et $\mathrm{al}^{30}$ reportan que más de $60 \%$ del total de individuos del sitio Azapa 140 del valle de Azapa en Arica presentan espina bífida oculta, como en los casos anteriores, se propone que la endogamia sería la principal causa de esta alta ocurrencia. En general esta mayor frecuencia de espina bífida se observa en poblaciones agro-alfareras y no así en poblaciones más tempranas ${ }^{30-32}$.

Considerando el efecto teratógeno del arsé- 
nico, este estudio plantea una posible relación entre altos niveles de arsénico en el ambiente de la quebrada de Camarones con la prevalencia de espina bífida oculta en la prehistoria. Por lo que se propone que existe una diferencia estadísticamente significativa entre la frecuencia de espina bífida oculta en el sacro de la muestra de Camarones versus la muestra control de Lluta y Azapa, en donde los niveles de arsénico ambiental, aunque elevados, se acercan más a la norma. Esta hipótesis ambiental difiere de las teorías de endogamia propuestas tradicionalmente como causa de espina bífida oculta y busca entregar nuevos antecedentes paleopatológicos al estudio de la problemática del arsénico ambiental en la zona.

\section{Material y Método}

Se analiza un total de 120 individuos prehispánicos, adultos mayores de 19 años de edad al morir, esqueletizados y parcialmente momificados de las colecciones bioantropológicas provenientes de los sitios arqueológicos: "Camarones 8" $(\mathrm{n}=16)$, “Camarones 9" $(\mathrm{n}=21)$, “Azapa 140" $(\mathrm{n}=73)$ y "Lluta 54 ” ( $\mathrm{n}=10)$, depositadas en el Museo Universidad de Tarapacá de Arica- San Miguel de Azapa.

La muestra de estudio proviene del sector costero de la quebrada de Camarones en una zona al sur de la desembocadura del río homónimo; el sitio Camarones 8 corresponde al período Intermedio Tardío (1100 al 1470 d.C.) y Camarones 9 al período Tardío (1470 al 1530 d.C.). La muestra control proviene del sector medio del valle de Azapa (Azapa 140) y el sector bajo del valle de Lluta (sitio Lluta 54). El sitio Azapa 140, corresponde al período Medio fase Maytas (700-1100 d.C.) e Intermedio Tardío fase San Miguel (1100-1470 d.C.); Lluta 54, corresponde al período Tardío
(1470-1530 d.C.). La muestra y muestra control fueron seleccionadas por su proveniencia geográfica y témporo-cultural, su disponibilidad para el estudio, por el buen estado de conservación y por los antecedentes previos de arsenicismo en Camarones $9^{2,33}$ y espina bífida oculta en Azapa $140^{30}$.

Como parte de la metodología aplicada, se determinó la edad biológica y el sexo de los individuos utilizando indicadores estándares ${ }^{34-36}$. Se realizó observación morfoscópica y radiológica a fin de detectar la presencia de espina bífida oculta a nivel sacro, el diagnóstico se consideró positivo cuando la falta de fusión del arco neural posterior ocurre al menos en la S1, S2 o S3, ya sea el segmento completo o incompleto.

Las radiografías fueron tomadas en el laboratorio de rayos X del Museo Universidad de Tarapacá de Arica- San Miguel de Azapa, por una asistente técnica capacitada (Leticia Latorre), en un equipo Shimadzu EZy-Rad en norma anteroposterior (AP), con el fin de diferenciar lo normal de lo patológico. Se contó además, con la cooperación del laboratorio de Rayos X de la Facultad de Ciencias de la Salud de la Universidad de Tarapacá.

Para analizar los datos se compararon las frecuencias de espina bífida oculta de la muestra y muestra control. Considerando que éstas son de tamaño reducido se comprobó la significancia estadística de los datos empleando la prueba exacta de Fisher a través del software MINITAB V15.

\section{Resultados}

La distribución de la población de la muestra y muestra control por sexo se observa en la Tabla 2. La muestra costera de Camarones 8 y 9 en conjunto, presenta una frecuencia de espina bífida oculta del 13,5\% registrándose cuatro casos en masculi-

Tabla 2. Distribución poblacional de adultos por sexo y sitio

\begin{tabular}{|lcccc|}
\hline Sexo & Camarones 8 & Camarones 9 & Azapa 140 & Lluta 54 \\
\hline Masculino & 5 & 11 & 25 & 5 \\
\hline Femenino & 9 & 9 & 0 & 5 \\
\hline Indeterminado & 2 & 1 & 73 & 0 \\
Total & 16 & 21 & 10 \\
\hline
\end{tabular}


Espina bífida oculta y su relación con arsénico en muestra prehispánica de Quebrada de Camarones - V. Silva-Pinto et al

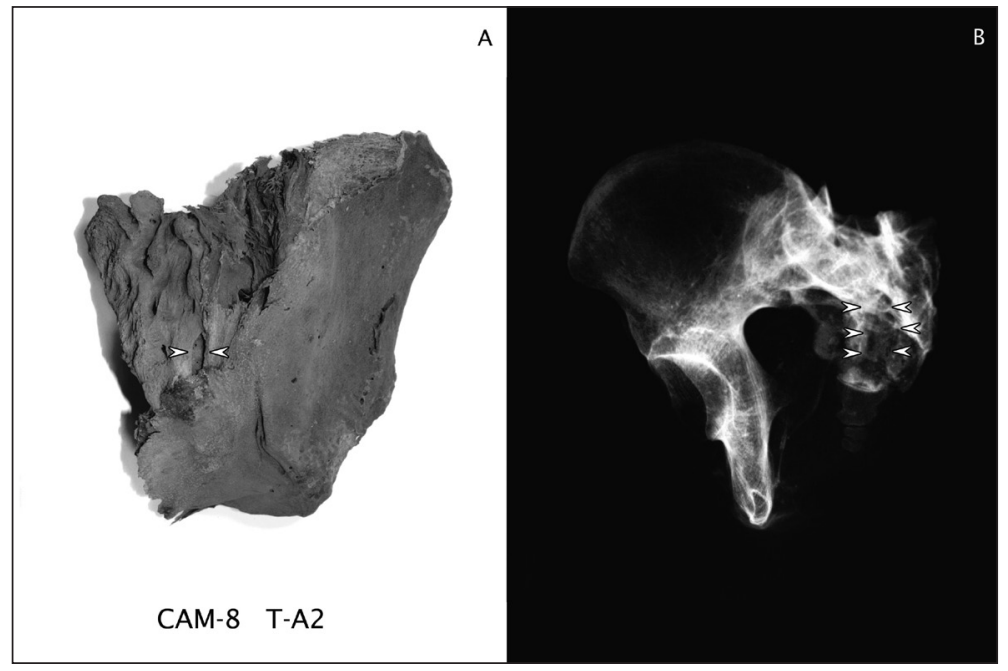

Figura 1. Espina bífida oculta sacra. Sitio Camarones 8 Tumba A2. Masculino, 3842 años de edad. A. Sacro y coxal derecho articulados vista posterior. B. Radiografía de sacro y coxal en vista antero-posterior.

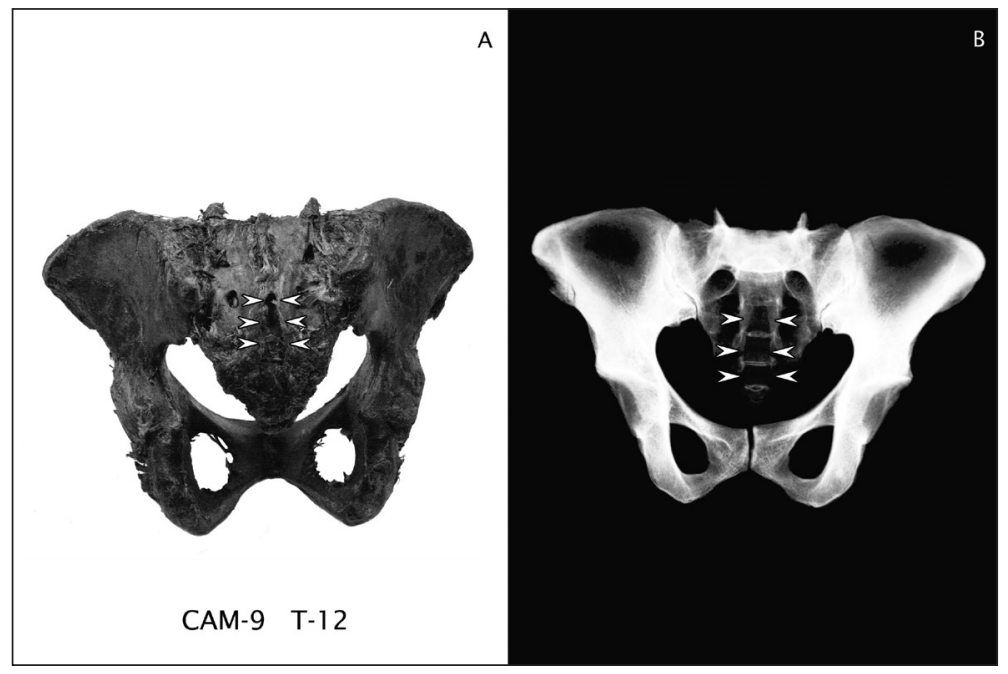

Figura 2. Espina bífida oculta sacra. Sitio Camarones 9 Tumba 12. Femenino, 35-40 años de edad. A. Pelvis articulada vista posterior. B. Radiografía de la pelvis en vista antero-posterior. nos y uno femenino (Figuras 1 a 3). En la muestra control proveniente de los valles de Lluta y Azapa se observó una frecuencia de $2,4 \%$, presentándose un individuo masculino y uno femenino afectados, ambos del sitio Azapa 140, lo que corresponde a 2,7\% del total de adultos del sitio. Uno de los casos presenta la patología con mayor severidad, ya que la falta de fusión del arco neural posterior afecta todos los segmentos sacros, incluida la quinta vértebra lumbar (Figura 4). En el gráfico de la Figura
5 se compara la frecuencia de espina bífida oculta entre la muestra y muestra control.

Los análisis estadísticos indican que la muestra costera (Camarones 8 y 9) presenta una frecuencia de espina bífida oculta en el sacro, casi seis veces mayor que la muestra control de los valles (Lluta 54, Azapa 140). El resultado de la Prueba Exacta de Fisher $\mathrm{p}=0,0284$ indica que hay diferencias estadísticamente significativas entre la muestra de Camarones y la muestra control de los valles. 


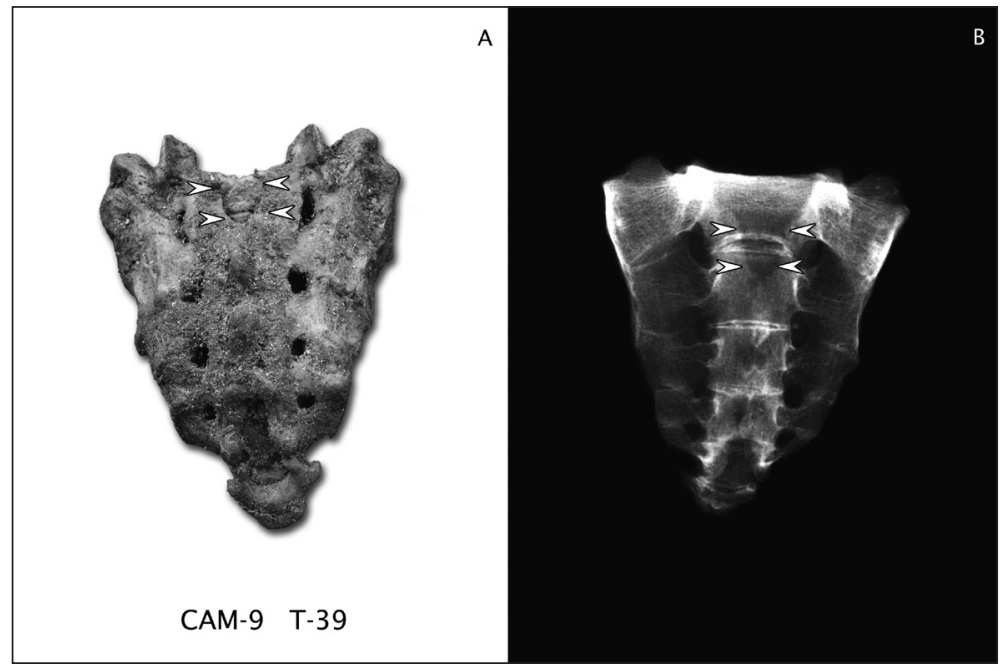

Figura 3. Espina bífida oculta sacra. Sitio Camarones 9 Tumba 39. Femenino, 35-40 años de edad. A. Hueso sacro vista posterior. B. Radiografía hueso sacro en vista antero-posterior.

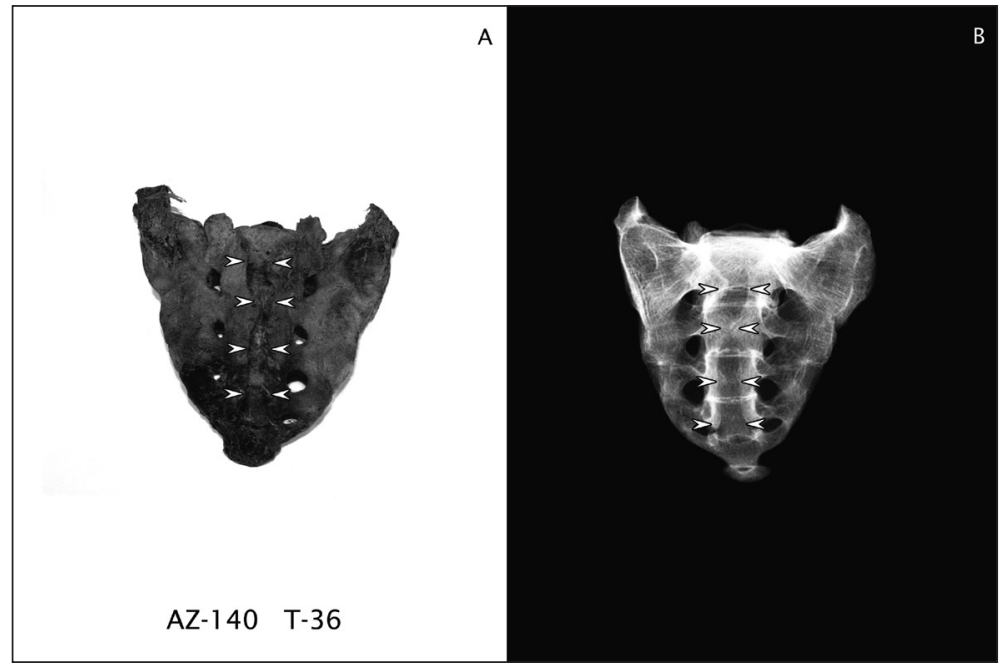

Figura 4. Espina bífida oculta sacra completa. Sitio Azapa 140 Tumba 36. Femenino, 19-22 años de edad. A. Hueso sacro vista posterior. B. Radiografía hueso sacro en vista antero-posterior.

\section{Discusión}

Se estima que, en la actualidad, alrededor de 8.000 personas del Norte de Chile (Regiones XV, I, II y III) se encuentran aún expuestas o afectadas por altas concentraciones de arsénico en el agua de bebida y los alimentos ${ }^{4}$. En la quebrada de Camarones se han tomado medidas sanitarias para mitigar los efectos del arsénico en la salud de la población, como la entrega de agua potable proveniente de Azapa a través de camiones aljibes. Estas medidas no logran ser suficientes para evitar que la población siga utilizando el agua local como agua de bebida, especialmente en áreas rurales y campamentos transitorios, además, de su utilización en el riego de los cultivos y bebida para los animales, incorporándose el arsénico a la cadena trófica e ingresando al organismo humano a través de los alimentos contaminados ${ }^{4-6}$.

La exposición crónica de la población actual de 


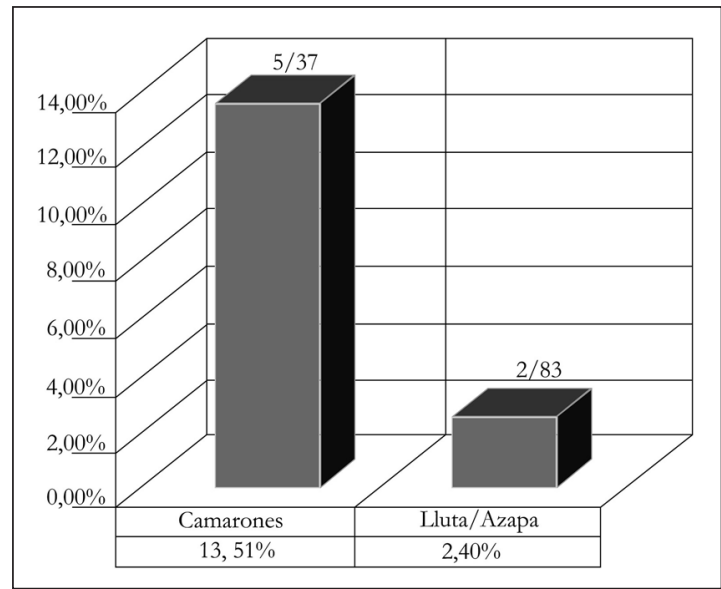

Figura 5. Gráfico comparativo de la frecuencia de espina bífida oculta entre la muestra en estudio (Camarones 8 y 9) y la muestra control (Lluta 54 y Azapa 140).

Camarones al arsénico ambiental, ha provocado el desarrollo de patologías como melanodermia, leucodermia o queratosis palmoplantar y otras afecciones atribuidas a arsenicismo crónico ${ }^{4-6}$. Debido a su origen natural, el arsénico se encuentra presente en los ecosistemas relacionados a la cordillera de Los Andes desde su formación inicial en la era terciaria y cuaternaria, por lo que representaría un problema atemporal ${ }^{2,6}$. En estudios multidisciplinarios previos (paleopatológicos y químicos) se detectaron afecciones a la piel relacionadas al arsenicismo crónico en momias naturales del sitio Camarones 9, comprobándose además, la presencia de altos niveles de arsénico en los tejidos y pelo de éstas y otras momias prehispánicas de Arica ${ }^{2,5,33}$. Se ha propuesto también arsenicismo crónico en momias naturales de Antofagasta, una región caracterizada por sus altos niveles de arsénico en el ambiente, lo que reflejaría que la contaminación ambiental por arsénico en la zona es un problema endémico que se remite al poblamiento temprano de estos ecosistemas hace más de 10.000 años ${ }^{37,38}$.

Ya sea por habitar en condiciones ambientales símiles o por parentesco genético entre poblaciones actuales y poblaciones prehispánicas, es posible a través de problemáticas actuales abordar problemas del pasado y viceversa. Por ello los estudios paleoepidemiológicos contribuyen al mejoramiento de las condiciones de salud de las poblaciones actuales ${ }^{32}$, al menos al dar una voz de alerta sobre las afecciones a la salud que vienen manteniéndose a través de los siglos, sin que se hayan implementado medidas sanitarias definitivas y no sólo paliativas.

Nuestros resultados indican una alta frecuencia de espina bífida oculta en la muestra costera de la quebrada de Camarones $(13,5 \%)$ y una baja frecuencia en la muestra control de los valles $(2,4 \%)$, con una diferencia estadísticamente significativa entre ellas. Creemos que la discrepancia entre nuestros resultados y lo reportado por Allison ${ }^{30}$ para el sitio Azapa 140, con más del 60\% de prevalencia de espina bífida oculta, se debe a diferencias en la metodología de diagnóstico, en nuestro caso no se consideró la presencia del hiato sacro como espina bífida, ya que éste es considerado un rasgo de variabilidad anatómica en las vértebras sacras S5 y S4-S5 $5^{39-41}$.

La muestra y muestra control provienen de sectores geográficos distintos, costa e interior de los valles respectivamente, pero son cronológicamente contemporáneos (700 a 1530 d.C.), durante este período las poblaciones humanas explotaban de forma eficiente los distintos pisos ecológicos, posibilitando la existencia de una economía mixta agro-marítima ${ }^{42-45}$. Este tipo de economía ha sido explicado a través del modelo de verticalidad de Murra $^{46}$, un modelo que ha sido fundamental en la arqueología, etnohistoria y antropología Andina y que habría permitido no sólo la explotación de recursos, sino también, la interacción entre los distintos grupos sociales existentes a través de mecanismos como el trafico caravanero y el establecimiento de lazos de parentesco entre las distintas etnias por medio del traslado de individuos de un grupo a otro ${ }^{44,45}$.

En base a lo anteriormente expuesto se analizan dos puntos importantes, por un lado la dieta podría estar influyendo en la menor frecuencia de espina bífida oculta en el valle de Lluta y Azapa, fundamentado en que el ácido fólico es considerado un elemento protector frente al desarrollo de malformaciones congénitas, en especial de defectos del cierre del tubo neural ${ }^{23}$, encontrándose en forma natural en algunos alimentos de origen americano como el poroto (Phaseolus vulgaris), la quínoa (Chenopodium quinua) y el maíz (Zea mays), por lo que el mayor consumo de éstos podría contribuir a una disminución de fallas en el cierre del tubo neural.

El segundo punto a analizar es la teoría de la endogamia o de la deriva génica en general, que 
explica la mayor frecuencia de espina bífida oculta por la reproducción entre miembros de una misma población de número reducido, que disminuye la variabilidad genética y aumenta la frecuencia de enfermedades hereditarias ${ }^{47}$.

Deficiencias nutricionales de la madre grávida o prácticas endogámicas no pueden descartarse como probables causas de una mayor ocurrencia de espina bífida oculta en la muestra de Camarones. Sin embargo, éstas se contradicen con el modelo de explotación e interacción social de los distintos pisos ecológicos propuesto para las poblaciones del Período Medio a Tardío en la prehistoria del Norte de Chile. Estos modelos y los resultados del presente estudio, refuerzan la hipótesis ambiental planteada y permiten proponer que el metaloide arsénico podría ser el principal agente ambiental causal de la mayor frecuencia de espina bífida oculta en las poblaciones prehispánicas de la quebrada de Camarones, lo que debiera ser evaluado a través de análisis epidemiológicos en poblaciones actuales de la región y en otras poblaciones prehispánicas.

Agradecimientos: Este trabajo fue apoyado por la Comisión Nacional de Investigación Científica y Tecnológica (CONICYT) de Chile, a través del proyecto FONDECYT No 1070575 y por la Línea de Bioarqueología del Convenio de Desempeño de la Universidad de Tarapacá UTA-MINEDUC, Arica Chile. Agradecemos en especial, la colaboración de Leticia Latorre y Carlos Ubeda por la toma de radiografías, a Octavio Lagos por la toma de fotografías, a Eugenio Aspillaga por sus recomendaciones al estudio, a Marcela Sepúlveda por el apoyo logístico, a Manuel Alarcón por la edición de las figuras y del texto, y a James Watson por la traducción del resumen al inglés.

\section{Referencias}

1. World Health Organization (WHO). Environmental health criteria for arsenic and arsenic compounds. Geneva: World Health Organization 2001; 224. Disponible en: http://www.who.int/ipcs/publications/ehc/ehc_224/ en/ [Consultado el 30 de noviembre de 2008].

2. Figueroa L. Arica inserta en una región arsenical: el arsénico en ambiente que la afecta y 45 siglos de arsenicismo crónico. Arica, Chile: Ediciones Universidad de Tarapacá 2001.
3. Castro M. Arsénico en el agua de bebida de América Latina y su efecto en la salud pública. Hojas de Divulgación Técnica. HDT - CEPIS Nº 95. 2004; p. 1-12. Disponible en: http://www.cepis.ops-oms.org/bvsair/e/hdt/hdt95/ hdt95.pdf [Consultado el 15 de diciembre de 2008].

4. Cornejo L, Mansilla HD, Arenas MJ, Flores M, Flores V, Figueroa L, et al. Remoción de arsénico en aguas del río Camarones, Arica, Chile utilizando la tecnología RAOS Modificada. En: Litter M. I., Jiménez G. A., editores. Avances en tecnologías económicas solares para desinfección, descontaminación y remoción de arsénico en aguas de comunidades rurales de América Latina (métodos FH y RAOS). Proyecto OEA AE 141. La Plata, Argentina 2004; p. 85-92. Disponible en: http://www.cnea.gov.ar/ xxi/ambiental/agua-pura/publicaciones/LibroBN.pdf [Consultado el 20 de diciembre de 2008].

5. Mansilla HD, Cornejo L. Chile. En: Litter M., editora. Relevamiento de comunidades rurales de América Latina para la aplicación de tecnologías económicas para la potabilización de aguas. Proyecto OEA AE 141/2001. CNEA, Buenos Aires 2002; 10-23. Disponible en línea en: http:// www.cnea.qov.ar/xxi/ambiental/agua-pura/3archivos/ relevamientol.pdf [Consulta: 13/05/2008].

6. Bundschuh J, Nicolli HB, Blanco M Del C, Blarasin M, Farías SS, Cumbal S, et al. Capítulo 7 Distribución de arsénico en la región sudamericana. En: Bundschuh J, Pérez A, Litter M, Editores. IBEROARSEN Distribución del arsénico en las regiones Ibérica e Iberoamericana. Editado por CYTED, Argentina 2008; 137-87.

7. Instituto Nacional de Normalización (INN). Chile, Norma Chilena 409/1. Of 2005, Agua Potable Parte 1: Requisitos, 2005.

8. World Health Organization (WHO). Guidelines for drinking water quality, Vol. 1: Recommendations. Ginebra, 1993.

9. European Union. Council Directive $98 / 83 /$ EC on the quality of water intended for human consumption. European Union, 1998.

10. Borgoño JM, Vicent P, Venturino H, Infante A. Arsenic in the drinking water of the city of Antofagasta: epidemiological and clinical study before and after the installation of a treatment plant. Environ Health Perspect 1977; 19: 103-5.

11. Centeno J, Mullick FG, Martínez L, Page N, Gibb H, Longfellow D, et al. Pathology related to chronic arsenic exposure. Environ. Health Perspect 2002; 110: 883-6.

12. Hopenhayn-Rich C, Browning S, Hertz-Picciotto I, Ferreccio C, Peralta C, Gibb H. Chronic arsenic exposure and risk of infant mortality in two areas of Chile. Environ Health Perspect 2000; 108: 667-73.

13. Hopenhayn C, Ferreccio C, Browning SR, Huang B, 
Peralta C, Gibb H, et al. Arsenic exposure from drinking water and birth weight. Epidemiology 2003; 14: 593602.

14. Posada J, Leyton V. Malformaciones congénitas. En: Montenegro MA, Mena M, Illanes J, Lemus D, editores. Embriología humana. Santiago, Ediciones Universidad de Chile 1999; 155-63.

15. Machado A, Hovland D, Pilafas S, Collins M. Teratogenic response to arsenite during neurulation: relative sensitivities of C57BL/6J and SWV/Fnn mice and impact of the splotch allele. J Toxicol Sci 1999; 51: 98-107.

16. Desesso J. Teratogen update: Inorganic arsenic. Teratology 2001; 63: 170-3.

17. Fem V. Arsenic as teratogenic agent. Environ Health Perspect 1977; 19: 215-7.

18. Martin LJ, Machado AF, Loza MA, Mao GE, Lee GS, Hovland Jr DN, et al. Effect of arsenite, maternal age, and embryonic sex on spina bifida, exencephaly, and resorption rates in the splotch mouse. Birth Defects Res A Clin Mol Teratol 2003; 67: 231-9.

19. Wlodarczyk B, Bennett G, Calvin J, Graig J, Finnel R. Arsenic-induced alterations in embryonic transcription factor gene expression: implications for abnormal neural development. Developmental Genetics 1996; 18: 306-15.

20. Nye J, Mclone D, Charrow J, Hayes E. Neural crest anomaly syndromes in children with spina bifida. Teratology 1999; 60: 179-98.

21. Nazer HJ, Cifuentes OL, Rodríguez CM, Rojas NM. Malformaciones del sistema nervioso central en el Hospital Clínico de la Universidad de Chile y maternidades chilenas participantes en el Estudio Colaborativo Latinoamericano de Malformaciones Congénitas (ECLAMC). Rev Med Chile 2001; 129: 1163-70.

22. Nazer HJ, López-Camelo J, Castilla EE. ECLAMC: Estudio de 30 años de vigilancia epidemiológica de defectos de tubo neural en Chile y en Latinoamérica. Rev Med Chile 2001; 129: 531-9.

23. López-Camelo JS, Orioli IM, Dutra M De G, NazerHerrera J, Rivera N, Ojeda ME, et al. Reduction of birth prevalence rates of neural tube defects after folic acid fortification in Chile. Am J Med Genet 2005; 135A: 120 5.

24. Turkel S. Congenital abnormalities in skeletal populations. En: Iscan MY, Kennedy K. Reconstructions of Life from the Skeleton. New York: A. R. Liss 1989; 109-27.

25. Aufderheide A, Rodríguez-Martin C. The Cambridge Encyclopedia of Human Paleopathology. Cambridge U.K. Cambridge University Press 1998.

26. Ferembach JMM. Frequency of spina bibida occulta in prehistoric human skeletons. Nature 1963; 199: 100-1.

27. Rodríguez-Martin C. Patología de la columna vertebral en poblaciones del pasado. Revisión de la población prehispánica de Tenerife. ERES-Serie de Arqueología 1995; 6: 157-70.

28. Mas-Pascual MA, González-Reimers E, Pérez E, Lugo MJ, Arnay-De-La-Rosa M, Velasco-Vázquez J, et al. Espina bífida en la población prehispánica del Hierro. Comunicaciones. Actas VII Congreso de la Asociación Española de Paleopatología 2003.

29. Gerszten PC, Gerszten E, Allison MJ. Diseases of the Spine in South American Mummies. Neurosurgery 2001; 48: 1: 208-13.

30. Allison M, Gerszten E, Fouant M. Paleopathology: Today's laboratory investigates yesterday's diseases. Diagnostic Med 1982; 5: 28-48.

31. Allison M. Informe biológico de la población Morro 1/6. En: Focacci G, Chacón S. Excavaciones arqueológicas en los faldeos del Morro de Arica, Sitios Morro 1/6 y 2/2. Chungará 1989; 22: 25-62.

32. Castro M, Aspillaga E. Paleopatología de las poblaciones prehistóricas. En: Rothhammer F. y Llop E. Poblaciones chilenas: Cuatro décadas de investigaciones bioantropológicas. Santiago, Editorial Universitaria 2004.

33. Figueroa L, Razmilic B, Allison M, González M. Evidencia de arsenicismo crónico en momias del valle de Camarones. Región de Tarapacá, Chile. Chungará 1988; 21:33-42.

34. Bass W. Human osteology: a laboratory and field manual. $4^{\text {a }}$ ed. Missouri, Archaeological Society Inc 1995.

35. Buikstra J, Ubelaker D. Editores. Standards for data collection from human skeletal remains. Arkansas, Archaeological Survey Research Series 44, 1994.

36. Ubelaker D. Human skeletal remains: Excavation, analysis, interpretations. $2^{\text {nd }}$ Edition. Washington: Taraxacum 1989.

37. Borgoño JM, Greiber R. Estudio epidemiológico del arsenicismo en la Ciudad de Antofagasta. Rev Med Chile 1971; 99: 702-7.

38. Arriaza B. An environmental hypothesis to explain the origin of Chinchorro artificial mummification. Chungará 2005; 37: 2: 255-60.

39. Albrecht T, Scutter S, Henneberg M. Radiographic method to assess the prevalence of sacral spina bifida occulta. Clinical Anatomy 2007; 20: 170-4.

40. Barnes E. Developmental defects of the axial skeleton in paleopathology. University Press of Colorado 1994.

41. Isidro A, Malgosa M. Paleopatología: La enfermedad no escrita. Elsevier España, 2003.

42. Chacama J. Integración Andina. En: Santoro C. et al. Pueblos del desierto. Ediciones Universidad de Tarapacá. Arica Chile 2001; 6: 52-64.

43. Santoro C. Caciques y control territorial. En: Santoro C. et al. Pueblos del desierto. Ediciones Universidad de 
Espina bífida oculta y su relación con arsénico en muestra prehispánica de Quebrada de Camarones - V. Silva-Pinto et al

Tarapacá. Arica Chile 2001; 7: 66-72.

44. Santoro C, Romero A, Standen V. Interacción social en los períodos intermedio tardío y tardío, valle de Lluta, norte de Chile. Manuscrito para ser publicado en la arqueología y la etnohistoria en los Andes, editado por J. Topic. 2003. Disponible en línea: http://www.uta.cl/ masma/yuta/pdf/Santoro_etal_Topic.pdf [Consultado el 25 de noviembre de 2008].

45. Schiapacasse V, Niemeyer H. Avances y sugerencias para el conocimiento de la prehistoria tardía en la desembo- cadura del valle de Camarones (Región de Tarapacá). Chungará 1989; 22: 63-84.

46. Murra J. El control vertical de un máximo de pisos ecológicos en la economía de las sociedades andinas. En: Visita de la Provincia de León de Huanuco en 1562, Murra J, editor. Universidad Nacional Hermilio Valdizán, Huanuco 1972; 427-76.

47. Curtis H, Barnes S, Schnek A, Flores G. Invitación a la biología. Sexta edición en español. Editorial Médica Panamericana 2007; Cap. 17: 256-70. 